\title{
Psychophysical assessment of magno- and parvocellular function in schizophrenia
}

\author{
Sandrine Delord ${ }^{1}$, Maria Giovanna Ducato ${ }^{2,3}$, Delphine Pins ${ }^{2,3}$, Frédéric Devinck ${ }^{4}$, Pierre \\ Thomas $^{2,3}$, Muriel Boucart ${ }^{2,3}$, and Kenneth Knoblauch ${ }^{5,6}$
}

1. Université Bordeaux 2, Equipe de Psychologie Cognitive, Laboratoire de Psychologie (EA 3662), , Bordeaux, France

2. CNRS FRE 2726, Laboratoire de Neurosciences Fonctionnelles et Pathologies, Lille, France

3. Université Lille 2, CHRU de Lille, Lille, France

4. Section of Neurobiology, Physiology and Behavior, Department of Ophthalmology, University of California-Davis, USA

5. INSERM, U371, Cerveau et Vision, Department of Cognitive Neuroscience, Bron, F69500, France

6. Université Claude Bernard Lyon 1, Lyon, France; IFR 19, Lyon France

Corresponding author

Sandrine Delord

Equipe de Psychologie Cognitive, Laboratoire de Psychologie (EA 3662),

U.F.R. des Sciences de l'Homme, Université Bordeaux 2,

3ter place de la Victoire

33076 BORDEAUX

France

Tel : (335) 57571978

Fax : (335) 57571977

sandrine.delord@psycho.u-bordeaux2.fr

Short title : Early processing in schizophrenia

Number of manuscript pages : 14

Number of tables : 1

Number of figures : 3 


\section{Psychophysical assessment of magno- and parvocellular function in schizophrenia}

Recently developed psychophysical techniques permit the biasing of the processing of the stimulus by early visual channels so that responses reflect characteristics of either magnoor parvocellular pathways (Pokorny \& Smith, 1997, J Opt Soc Am A, 14, 2477). We used such techniques to test psychophysically whether the global magnocellular dysfunction reported in schizophrenia also affects early processes, as well. Seven schizophrenic patients and 19 normal controls participated. The task was a 4-alternative forced-choice luminance discrimination, using a $2 \times 2$ configuration of four 1 deg squares. Target luminance threshold was determined in 3 conditions: the stimulus, including the target, was pulsed for $17 \mathrm{~ms}$ (pulse paradigm), the target was presented on a steady background of 4 squares (steady paradigm), or the target was presented alone (no background paradigm). We replicated previous results demonstrating magnocellular and parvocellular signatures in control participants. No evidence for an early magnocellular deficit could be detected as the thresholds of all schizophrenic observers were higher both in the steady paradigm (presumed magnocellular mediation), as well as in the pulse paradigm (presumed parvocellular mediation). Magnocellular dysfunction, if present in schizophrenia, must concern more integrated processes, possibly at levels at which parvocellular and magnocellular paths interact.

Keywords : psychophysics, magnocellular, parvocellular, schizophrenia 


\section{Introduction}

While schizophrenia is a cognitive disorder, there have been a number of reports of visual processing impairment, typically associated with the magnocellular and/or dorsal streams (e.g., Butler \& Javitt, 2005; Schwartz et al., 2001). The exact level of the impairment, however, is uncertain. Electrophysiological studies suggest an impairment as early as primary visual cortex. For example, visual evoked potentials (VEP) recorded over the occipital lobe displayed a lower overall response for schizophrenic patients relative to normal volunteers, specifically when the visual stimuli biased processing toward a magnocellular pathway (Butler, et al., 2001). In addition, analysis of steady-state VEP spectra indicated attenuation of the second harmonic response with little effect on the fundamental at low spatial frequencies (Kim, et al., 2005). The authors suggested a deficit in magnocellular signals to account for this result, based on evidence of their contribution to the second harmonic response. In contrast, VEPs measured during a higher level task involving identification of fragmented forms revealed an impairment within the Lateral Occipital Complex (LOC) via crossover input from the dorsal stream (Doniger, et al., 2002). This result was taken to suggest that initial stages of ventral stream processing are intact but that the deficit results from an abnormal interaction between dorsal (magno-dominated) and ventral pathways. Moreover, Event Related Potentials revealed no decreases in early components, associated with sensory processing, in schizophrenia (van der Stelt, et al., 2004).

Functional imaging has indicated activation differences between patients and controls in cortical extrastriate area MT (Braus et al. 2002). Similarly first-degree non-psychotic relatives of persons with schizophrenia showed a right hemisphere impairment in MT (Bedwell, et al., 2004). These studies do not, however, exclude that magnocellular-like deficits could be generated pre-cortically. Interestingly, somatosensory impairments in schizophrenia, observed within $20 \mathrm{~ms}$ of target presentation, are interpreted in terms of a thalamic dysfunction which 
interferes with the flow of information from sensory input to cortex (Waberski, et al., 2004).

Psychophysical approaches to this question have been limited principally to studies of backward masking and contrast sensitivity. In masking studies, spatial and timing differences in the stimuli are exploited to probe visual coding mechanisms (Breitmeyer \& Ogmen, 2000). Generally speaking, masking phenomena can be well explained in terms of the dynamics of early coding mechanisms even when target processing depends on a high level task like identification (Delord, 1998). Nevertheless, alternative hypotheses in terms of attentional allocation are difficult to exclude (Enns \& Di Lollo, 2000). Masking studies have systematically revealed abnormalities in schizophrenics (Butler \& Javittt, 2005; Cadenhead et al., 1998; McClure, 2001; Schwartz, et al., 2001), though differences in stimulus conditions, such as stimulus onset asynchrony (Grandhom \& Verney, 2004; Slaghuis, 2004) and spatial and chromatic characteristics of the mask (Bedwell, et al., 2002; Butler, et al., 2002; Schechter, et al., 2003) have led to conflicting results and interpretations as to the selectivity and the level of deficit. Contrast sensitivity measurements have not indicated unequivocally either whether schizophrenics show spatial frequency selective losses (Chen et al., 1999; Keri et al., 2002; Slaghuis, 2004). Chen et al. (2003) suggest that inconsistencies between reports can be attributed to secondary effects related to medication that may differ between studies.

The present work is aimed at examining psychophysically the status of early visual coding in schizophrenia. For this purpose, we used the short version of the steady and pulse paradigms introduced by Pokorny and Smith (1997) that was adapted to testing patients (Alexander et al., 2001). The tests exploit the different contrast gain characteristics of the parvocellular (P) and magnocellular (M) pathways. The high contrast gain in the M pathway results in high luminance contrast sensitivity at low contrasts but rapid loss of sensitivity to contrast differences as contrast increases due to response saturation. Conversely, the $\mathrm{P}$ pathway is less sensitive at low contrasts but its low contrast gain leaves its luminance 
contrast sensitivity relatively intact at contrast levels that saturate the M pathway signals. These gain differences in the pathways, already evident in the retinal output (Kaplan \& Shapley, 1986), permit the design of stimuli that bias processing so that responses depend more on signals transmitted through one or the other pathway.

\section{Methods}

\section{Participants}

The research followed the tenets of the Declaration of Helsinki and informed consent was obtained from all participants. Seven schizophrenic patients and 19 normal controls participated in the experiment. The patients were in-patients meeting DSM-IV criteria for schizophrenia admitted at the psychiatric departments of Lille, Amiens and Armentières (France). They ranged in age from 18 to 52 years. Diagnosis of schizophrenia was defined by DSM-IV on the basis of the mini International Neuropsychiatric Interview plus (M.I.N.I. plus, Sheehan et al., 1998), a short diagnostic structured interview assessed by an experienced clinician. The Positive and Negative Syndrome Scale (PANSS) was used to measure the severity of psychopathology (Kay, et al., 1987). Nineteen healthy volunteers, ranging from 18 to 54 years, were recruited. All volunteers were paid for their participation. Participants were checked for visual acuity, color vision (Ishihara test) and contrast sensitivity. Any of the following criteria excluded a participant from the study, either patients or controls: ophthalmic and/or neurological disease, uncorrected myopia or mental retardation. The patient and the control groups did not significantly differ in gender (controls: 10M/9F; patients: $\left.5 \mathrm{M} / 2 \mathrm{~F} ; \chi^{2}=0.17, \mathrm{p}=0.68\right)$ nor in age (mean $(\mathrm{sd})$ : controls 27 (9.5); patients 35 (15.1) $; \mathrm{t}(24)=-1.66, \mathrm{p}=0.11)$. Demographic data and medication of the patients are presented in Table 1.

Insert Table 1 about here 


\section{Apparatus}

The experiment was conducted inside the hospital and testing of the patients was supervised by a medical practitioner. All stimuli were presented on a Nokia 445 pro color monitor (21') driven by software on a PC Hewlet Packard 128 Mo de RAM Pentium III 1 GB under the control of an Intel 82815 Graphics card that provides 8 bits of resolution for each gun of the $640 * 480$ display. The screen was run at a field rate of $75 \mathrm{~Hz}$, non-interlaced. The voltage-phosphor luminance relationship was linearized with look-up tables. Calibration of the screen was performed with a Minolta CS100 chromameter. Stimulus generation and presentation were performed using Matlab 5.0 (The MathWorks) and the MatVis toolbox (Carney, 1990). The observer was placed $57 \mathrm{~cm}$ (with a chinrest) from the display in an otherwise dimly illuminated room. The background of the screen was set at $14.9 \mathrm{~cd} / \mathrm{m}^{2}$.

\section{Stimuli}

The stimulus consisted of a $2 \times 2$ array of 4 squares of side 1 deg, each one separated by 3 min and arranged around a central fixation point (Figure 1). The four squares were either higher $\left(17.9 \mathrm{~cd} / \mathrm{m}^{2}\right.$, i.e., $\left.+20 \%\right)$, lower $\left(11.9 \mathrm{~cd} / \mathrm{m}^{2}\right.$, i.e., $\left.-20 \%\right)$ or equal in luminance to the background. On each trial, a randomly chosen square was slightly varied in contrast relative to the others (always a luminance increment).

Insert Figure 1 about here

\section{Procedure}

Figure 1 shows the spatial and temporal configuration of the 5 experimental conditions. When the pedestals were present, the temporal sequence varied : either pulsed briefly (pulse paradigm), or continuously presented (steady paradigm). The experimental session lasted from 45-90 min and comprised 5 blocks, one for each condition (pulse-brighter pedestals, pulse-darker pedestals, zero-pedestals, steady-brighter pedestals, steady-darker 
pedestals). The conditions were presented in a random order different for each participant. For each block, the contrast threshold was first approximated with a descending method of limits, and then calculated using the method of constant stimuli with 6 levels of contrast (equally spaced on a log scale) individually adjusted on the basis of the results obtained with the method of limits. Threshold was defined from a Weibull function fit to the data as the contrast generating a probability of 0.72 of correct response.

In the zero-pedestal condition, the target square was presented alone following the presentation of a $500 \mathrm{msec}$ fixation point. The task was to locate the target by pressing the appropriate key $(1,2,4$, or 5 of the numeric pad). In the two pulse paradigms, the stimulus was presented for $17 \mathrm{msec}$, together with an array of three square-pedestals and the task was to locate the square among the four that appeared brighter than the others. In the two steady paradigms, the stimulus was preceded and followed by a four pedestal screen that lasted for $500 \mathrm{msec}$.

\section{Results}

Figure 2 shows the average thresholds for the 5 experimental conditions for each group. Five observers ( 4 controls and 1 patient) did not complete one condition because of time constraints or fatigue. The solid lines, corresponding to the controls, replicate closely the results obtained by Alexander et al. (2001). A monotonically increasing function is found for the steady condition (thin line) and a V-shaped function for the pulse condition (thick lines). Note that the zero pedestal condition experimentally is common to both pulsed and steady pedestal conditions since both reduce to this condition at zero contrast. The pulse condition lines have typically not been connected through this point, however, to emphasize that the zero pedestal condition is mediated theoretically by the same pathway that mediates the steady condition (Pokorny \& Smith, 1997). The average thresholds for the schizophrenic 
group (dashed lines, thin and thick for steady and pulsed pedestals, respectively) tend to follow those of the controls though they are higher and fall outside the $95 \%$ confidence intervals for all conditions, indicated as error bars on the control points.

\section{Insert Figure 2 about here}

A mixed-effects analysis of variance (anova) was performed with group (Control, Schizophrenic) and experimental condition as fixed effects and subject as a random effect. A significant effect of condition was found $(F(4,91)=145.0, p<0.001)$. Comparisons of the means based on contrasts indicated that in the steady condition, the difference in thresholds between the negative and zero pedestals was not significant $(\mathrm{t}(91)=-0.001, \mathrm{p}=0.99)$, but the difference between the positive and zero pedestals was $(\mathrm{t}(91)=8.8, \mathrm{p}<0.001)$. In the pulsed condition, the difference of both negative and positive pedestals from the zero pedestal was significant (negative: $\mathrm{t}(91)=10.8, \mathrm{p}<0.001$; positive: $\mathrm{t}(91)=16.6, \mathrm{p}<0.001$ ). The effect of group was also significant $(\mathrm{F}(1,24)=20.1, \mathrm{p}<0.001)$ but the interaction between group and condition was not $(\mathrm{F}(4,91)=0.99, \mathrm{p}=0.41)$.

Insert Figure 3 about here

Figure 3 shows the average control data (thick lines) compared with the data from the individual patients for the steady (top) and pulsed (bottom) pedestal conditions (thin lines). The greater variability of the patients is evident here indicating that some of the patients' thresholds are close to the average normal data. One patient showed a V-shaped function for the steady condion (inverted triangles in Figure 3, top) and another a monotonic function for the pulsed condition (x's in Figure 3, bottom). Nevertheless, across all conditions, over 70\% 
of the patient observations fall above the upper $95 \%$ confidence limit of the control data.

\section{Discussion}

In normal observers, the steady and pulsed pedestal paradigms generate threshold contours which vary in a manner consistent with the characteristics of parvo- and magnocellular contrast gain control mechanisms (Pokorny \& Smith, 1997). The steady pedestal condition generates thresholds that vary monotonically with luminance. It is argued that the discrimination in this case depends on the comparison of the spatial differences between the four stimulus fields (Smith \& Pokorny, 2003). These are low contrast and, thus, would favor discrimination based on signals in the magnocellular pathway that is sensitive to small contrast differences. In contrast, the pulsed-pedestal is proposed to briefly saturate the high gain mechanisms in the magnocellular pathway and produces a $\mathrm{V}$-shaped function as the discrimination switches to being constrained by the low-gain parvocellular pathway at high pedestal contrasts.

Schizophrenics show qualitatively similarly shaped functions to normal controls. As the shape of these functions is thought to reflect the properties of contrast gain control mechanisms imposed at the retinal level (Smith \& Pokorny, 2003), our results argue against a low level deficit in visual processing. The relative heights of the curves are proposed, instead, to reflect summation processes over populations of cells, mediated upstream from the retina, possibly at a cortical level (Smith \& Pokorny, 2003). We found that the thresholds of the schizophrenic group were significantly higher than those of the control group. This difference could reflect a simple criterion difference between the two groups or alternatively a difference in post-retinal processing. As the interaction between group and experimental condition was not significant, the differences between groups affect all conditions equally, consistent with a criterion effect. Hence, the lack of significant interaction argues against a deficit that is selective for one pathway. 
Another possibility is that the raised thresholds are secondary effects of the patients' medication. This is difficult to assess directly because, on the one hand, it would be unethical to withhold medication but, on the other hand, unreasonable to test patients when in an acute phase, just prior to receiving medication. Benzodiazepines have been reported to produce overall losses of sensitivity (Blin, et al., 1993 ; Giersch, et al., 1997). Long term use of benzodiazepines, however, seems to produce little effect on retinal function (Stafanous et al., 1999). Chen et al. (2003) found that typical antipsychotic drugs raised contrast thresholds while atypical ones did not. Consistent with these findings, two patients from Table 1 (DN and SS) with very different dosages of atypical neuroleptics displayed quite similar thresholds (open circles and diamonds, respectively, from Figures $3 \mathrm{a}$ and $\mathrm{b}$ ).

Some caveats must be raised, however. The sample size examined here was small. This reflects partly the difficulty of finding patients on whom the extended testing required to estimate thresholds would be possible. The data are heterogeneous which might, also, reflect the small sample size or, alternatively, might be due to real sources of variability in the schizophrenic population (Holcomb, et al., 2004; Wexler, et al., 2003). It would be desirable to perform more extensive examinations of the most psychophysically extreme patients, when possible, in future studies.

In conclusion, our data argue against an early magnocellular dysfunction in schizophrenia. If a magnocellular dysfunction is present, it may concern post-retinal processes, possibly at the level of parvo- and magnocellular interactions in the cortex.

\section{Acknowledgments}

This research was supported by a grant (Action Concertée Incitative : Temps et Cerveau) from the French ministry of national education and research. The authors are grateful to Stéphane Thimel for his assistance. 


\section{References}

Alexander, K.R., Pokorny, J., Smith, V.C., Fishman, G.A., \& Barnes, C.A. (2001). Contrast discrimination deficits in retinis pigmentosa are greater for stimuli that favor the magnocellular pathways. Vision Research, 41, 671-683.

Bedwell J.S., Brown J.M., \& Miller L.S. (2002). The magnocellular visual system and schizophrenia: what can the color red tell us? Schizophrenia Research, 63, 273-284.

Bedwell, J.S., Miller, L.S., Brown, J.M., McDowell, J.E., \& Yanasak, N.E. (2004). Functional magnetic resonance imaging examination of the magnocellular pathway in non psychotic relatives of persons with schizophrenia. Schizophrenia Research, 71, 509-510.

Blin, O., Mestre, D., Paut, O, Vercher, J.L., \& Auderbert, C. (1993) GABA-ergic control of visual perception in healthy volunteers: effects of midazolam, a benzodiazepine, on spatial-temporal contrast sensitivity. British Journal of Clinical Pharmacology, 36, 117 124.

Breitmeyer B.G., \& Ogmen H. (2000). Recent models and findings in visual backward masking: a comparison, review, and update. Perception \& Psychophysics, 62, 1572-1595.

Butler P.D., DeSanti L.A., Maddox J., Harkavy-Friedman J.M., Amador X.F., Goetz R.R., Javitt D.C., \& Gorman J.M. (2002). Visual backward-masking deficits in schizophrenia: relationship to visual pathway function and symptomatology. Schizophrenia Research, 59. 199-209.

Butler, P.D., \& Javitt, D.C. (2005). Early-stage visual processing deficits in schizophrenia. Current Opinion in Psychiatry, 18, 151-157.

Butler, P.D., Schechter, I., Zemon, V., Schwartz, S.G., Greenstein, V.C., Gordon, J.G., Schroeder, C.E., \& Javitt, D.C. (2001). Dysfunction of early-stage visual processing in schizophrenia. American Journal of Psychiatry, 158, 1126-1133.

Braus, D.F., Weber-Fahr, W., Tost, H., Ruf, M., Henn, F.A. (2002). Sensory information 
processing in neuroleptic naïve first-episode schizophrenic patients : a functional magnetic resonance imaging study. Archives of General Psychiatry, 59, 696-701.

Cadenhead, K.S., Serper, Y., \& Braff, D.L. (1998) Transient and sustained visual backward masking deficits of schizophrenia patients. Biological Psychiatry, 43, 132-138.

Carney, T. (1990). http://www.neurometrics.com/MatVisCommands/MatVisHome.htm. MatVis. Oakland (USA): Neurometrics Institute.

Chen, Y., Levy, D.L., Sheremata, S., Nakayama, K., Matthysse, S. \& Holtzman, P.S. (2003) Effects of typical, atypical and non antipsychotic drugs on visual contrast detection in schizophrenia. American Journal of Psychiatry, 160, 1795-1801.

Chen, Y., Palafox, G., Nakayama, K., Levy, D., Matthysse, S., \& Holtman, P.S. (1999). Motion perception in schizophrenia, Archives of General Psychiatry, 56, 145-154.

Delord, S. (1998). Which mask is the most efficient: A pattern or a noise? It depends on the task. Visual Cognition, 5, 313-338.

Doniger, G.M., Foxe, J.J., Murray, M.M., Higgins, B.A., \& Javitt, D.C. (2002). Impaired visual object recognition and dorsal/ventral stream interaction in Schizophrenia. Archives of General Psychiatry, 59, 1011-1020

Enns, J.T., \& Di Lollo, V. (2000) What's new in visual masking? Trends in Cognitive Science, 4, 345-352.

Giersch, A., Boucart, M., \& Danion, J.-M. (1997) Lorazepam, a benzodiazepine, induces atypical distractor effects with compound stimuli: A role for line-ends in the processing of compound letters. Visual Cognition, 4, 337-372.

Granholm E., \& Verney S.P. (2004) Pupillary responses and attentional allocation problems on the backward masking task in schizophrenia. International Journal of Psychophysiology, 52, 37-51

Holcomb, H.H., Parwani, A., McMahon, R.P., Medoff, D.R., Frey, K., Lahti, A.C., \& 
Tamminga, C.A., (2004). Parametric study of accuracy and response time in schizophrenic persons making visual or auditory discriminations. Psychiatry Research, $\underline{127}, 207-216$.

Kay, S.R., Fiszbein, A., \& Opler, L.A. (1987). The Positive and Negative Syndrome Scale (PANSS) for schizophrenia. Schizophrenia Bulletin, 13, 261-276.

Kaplan, E. \& Shapley, R. M. (1986) The primate retina contains two types of ganglion cells, with and high and low contrast sensitivity. Proceedings of the National Academy of

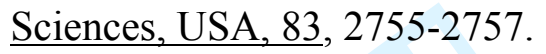

Keri S., Antal A., Szekeres G., Benedek G., \& Janka Z.( 2002) Spatiotemporal visual processing in schizophrenia. Journal of Neuropsychiatry and Clinical Neuroscience, 14 , 190-196.

Kim, D., Zemon, V., Saperstein, A., Butler, P.D., \& Javitt. D.C. (2005). Dysfunction of earlystages visual processing in schizophrenia : harmonic analysis. Schizophrenia Research, $\underline{76}, 55-65$.

McClure R.K. (2001). The visual backward masking deficit in schizophrenia. Progress in Neuropsychopharmacology and Biological Psychiatry, 25, 301-311.

Pokorny, J., \& Smith, V.S. (1997). Psychophysical signatures associated with magnocellular and parvocellular pathways contrast gain. Journal of the Optical Society of America A, 14, 2477-2486.

Schechter, I., Butler, P.D., Silipo, G., Zemon, V., \& Javitt, D.C. (2003). Magnocellular and parvocellular contributions to backward masking dysfunction in schizophrenia. Schizophrenia Research, 64, 91-101.

Schwartz, B.D., Tomlin, H.R., Evans, W.J., \& Ross, K.V. (2001). Neurophysiologic mechanisms of attention: a selective review of early information processing in 
schizophrenics. Frontiers in Bioscience, 6, 120-134.

Sheehan, D.V., Lecrubier, Y., Sheehan, K.H., Amorim, P., Janavs, J., Weiller, E., Herguetta, T., Baker, R., \& Dunbar, G.C., (1998). The Mini-International Neuropsychiatric Interview (M.I.N.I.) : the development and validation of a structured diagnostic psychiatric interview for DSM-IV and ICD-10. Journal of Clinical Psychiatry, 59, suppl 20:22-33; quiz 34-57.

Slaghuis, W. L. (2004). Spatio-temporal luminance contrast sensitivity and visual backward masking in schizophrenia. Experimental Brain Research, 156, 196-211

Smith, V. C., \& Pokorny J. (2003). Pyschophysical correlates of parvo- and magnocellular function. In Normal and Defective Colour Vision, Eds :. Mollon, J.D., Pokorny, J. \& Knoblauch, K., pp. 91-105, Oxford, England: Oxford University Press.

Stefanous, S.N., Clarke, M.P., Ashton, H. \& Mitchell, K.W. ((1999) The effect of long-term use of benzodiazepines on the eye and the retina. Documenta Ophthalmologica, 99, 55-68. van der Stelt, O., Frye, J., Lieberman, J.A., \& Belger, A. (2004). Impaired P3 generation reflect high-level and progressive neurocognitive dysfunction in Schizophrenia. Archives of General Psychiatry, 61, 237-248.

Waberski, T.D., Norra, C., Kawohl, W., Thyerlei, D., Hock, D., Klostermann, F., Curio, G., Buchner, H., Hoff, P., \& Gobbelé, R. (2004). Electrophysiological evidence for altered early cerebral somatosensory signal processing in schizophrenia. Psychophysiology, 41, 361-366.

Wexler, B.E., Nicholls, S.S., \& Bell, M.D. (2003). Instability of cognitve processing systems in schizophrenia. Schizophrenia Research, 71, 513-514. 


\section{Figure captions}

Table 1: Patient summary information.

Figure 1: Examples of the five experimental conditions used in the experiment: the two pulsed paradigms are shown on the left, with the pedestals brighter or darker than the background and the randomly placed target is upper left or lower right, respectively; the zero pedestal paradigm is shown in the center (target on the upper right), and the two steady pedestal paradigm are on the right, with the 3 pedestals brighter or darker than the background.

Figure 2: Mean results: mean incremental contrast thresholds as a function of the pedestal contrast. Solid lines and open symbols show results for control observers and dashed lines and black symbols for the schizophrenic patients. Thick lines and circles are used for the pulse condition and thin lines and squares for the steady condition. Error bars represent $95 \%$ confidence limits for the mean of the controls. For variability of the patients, see Figure 3.

Figure 3: The mean results of the control group (thick lines with black circles) are compared with the individual patients (thin lines) for steady (top) and, pulsed (bottom) pedestals. 
Table 1

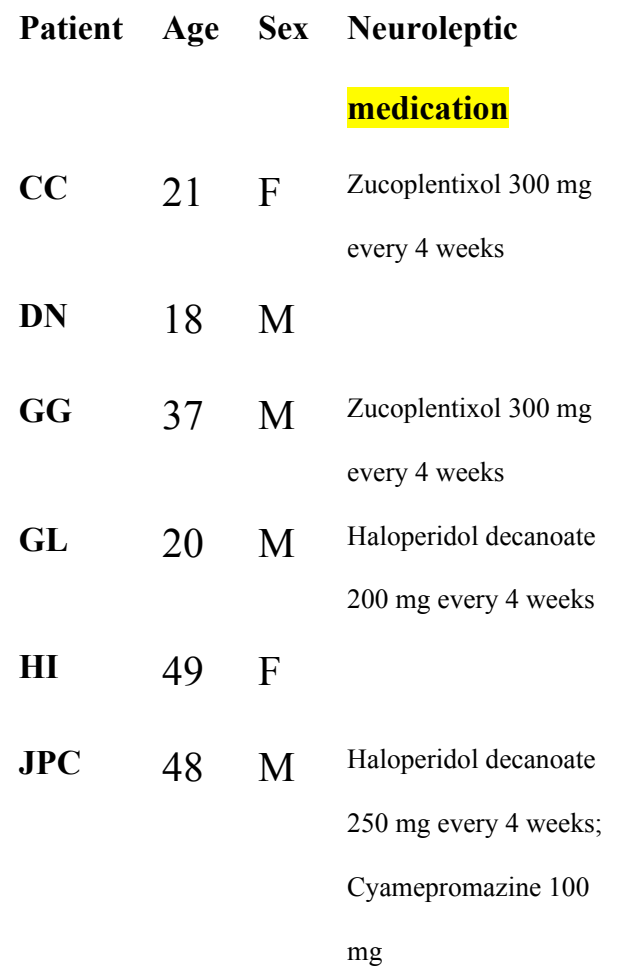

\begin{tabular}{|c|c|c|c|c|c|c|}
\hline Atypical neuroleptic & Chlorpromazine & Benzodiazepines & PANSS & PANSS & PANSS & PANSS \\
\hline \multirow{2}{*}{ medication } & equivalence & & positive scale & negative scale & general scale & Total \\
\hline & $600 \mathrm{mg}$ & & 21 & 16 & 37 & 74 \\
\hline \multirow[t]{3}{*}{ Risperidone 4mg } & $400 \mathrm{mg}$ & Lorazepam $7 \mathrm{mg}$ & 26 & 32 & 59 & 117 \\
\hline & $600 \mathrm{mg}$ & & 18 & 35 & 49 & 102 \\
\hline & $400 \mathrm{mg}$ & Lorazepam $5 \mathrm{mg}$ & 23 & 16 & 39 & 78 \\
\hline \multirow[t]{2}{*}{ Clozapine $250 \mathrm{mg}$} & $250 \mathrm{mg}$ & Lorazepam $1 \mathrm{mg}$ & 33 & 29 & 56 & 118 \\
\hline & $600 \mathrm{mg}$ & Lorazepam $7.5 \mathrm{mg}$ & 26 & 32 & 56 & 114 \\
\hline Amisulpride $1200 \mathrm{mg}$ & $1200 \mathrm{mg}$ & Clorazepate $150 \mathrm{mg}$ & 20 & 21 & 51 & 92 \\
\hline
\end{tabular}


Figure 1

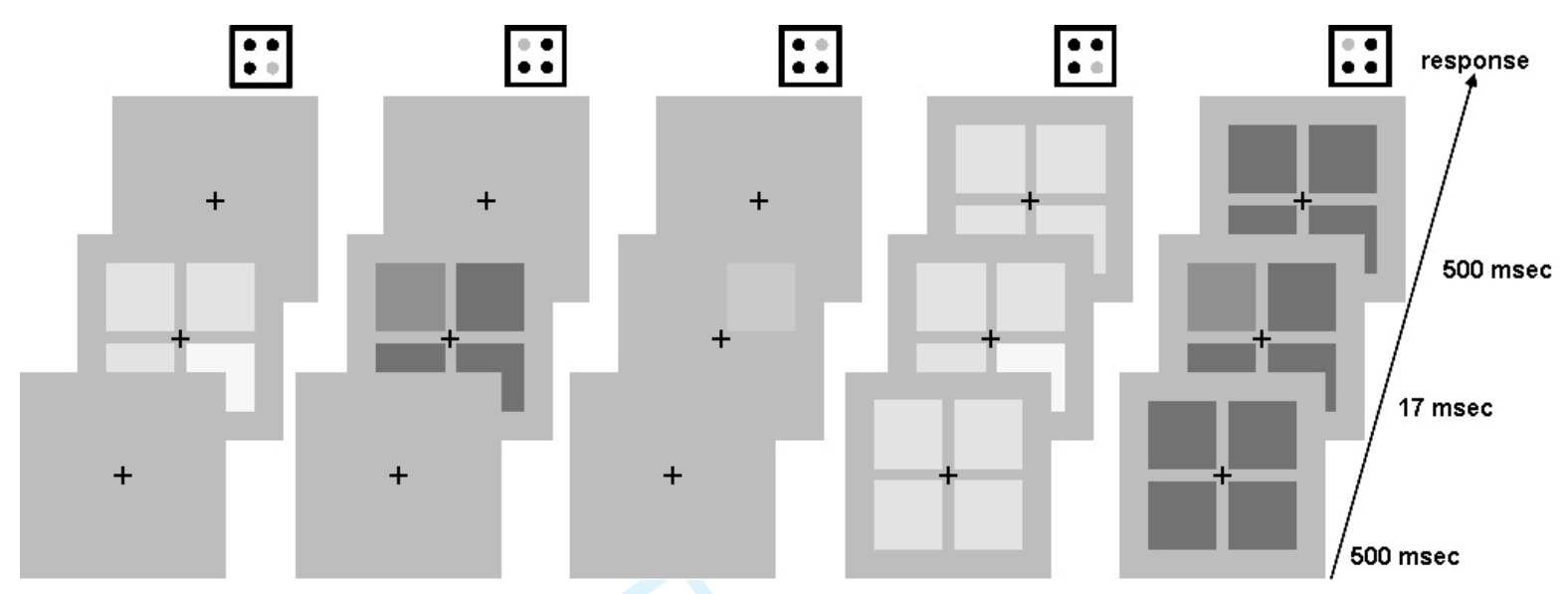




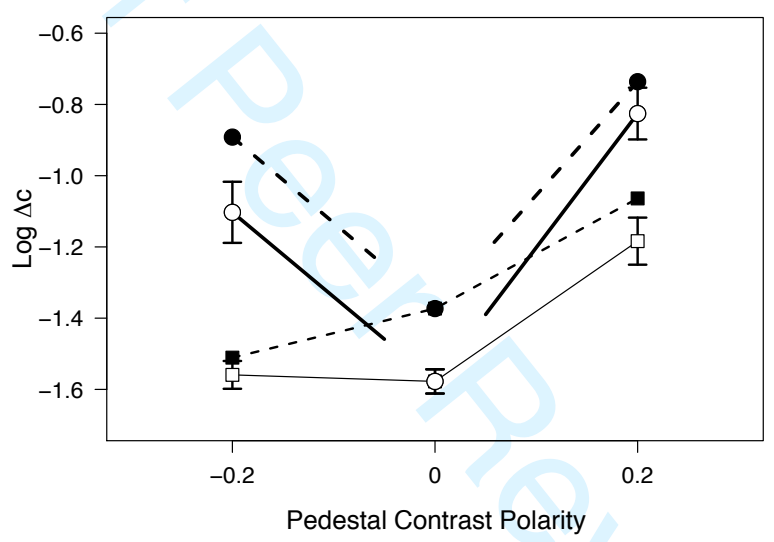



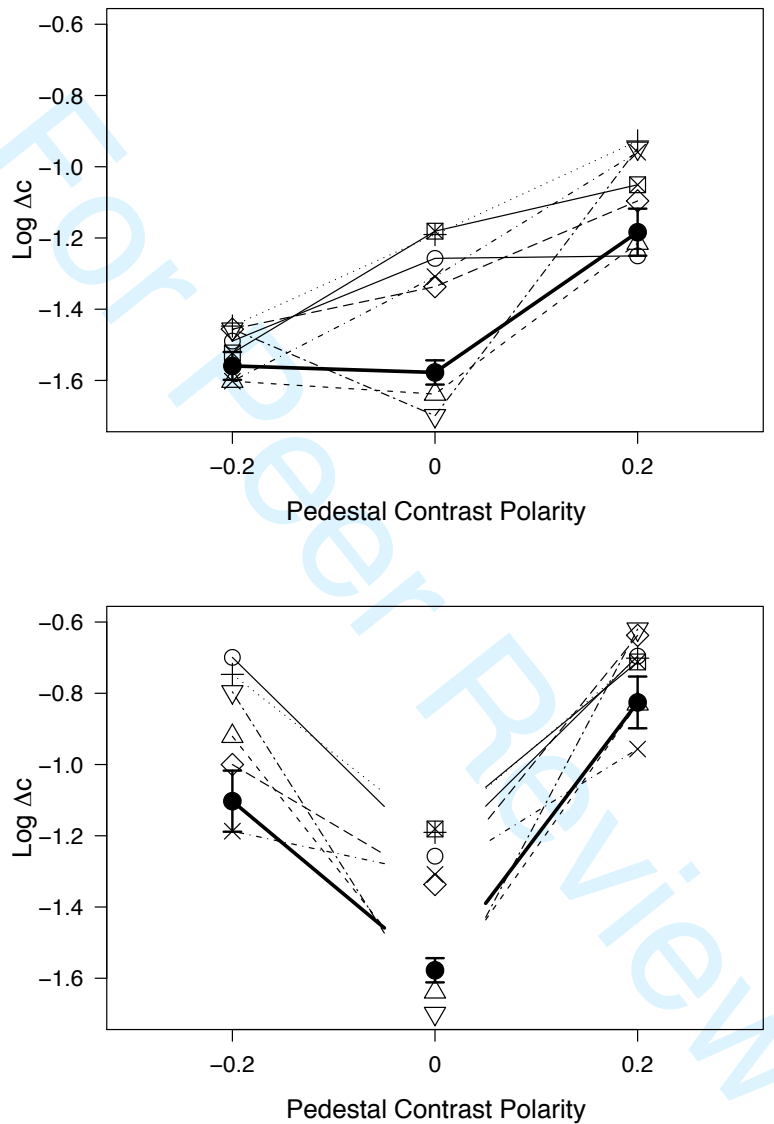